\title{
Avaliação do cuidado à saúde da gestante no contexto do Programa Saúde da Família
}

\author{
Evaluation of the care to the pregnant woman's health \\ in the context of the Family H ealth Program
}

\author{
Glauce Dias da Costa ${ }^{1}$ \\ Rosângela M inardi M itre Cotta ${ }^{1}$ \\ JoséRoberto Reis ${ }^{2}$ \\ Rodrigo Siqueira-Batista ${ }^{3}$ \\ Andréia Patrícia Gomes ${ }^{3}$ \\ Sylvia do Carmo Castro Franceschini ${ }^{1}$
}

${ }^{1}$ Departamento deN utrição eSaúde, Universidade Federal deViçosa. Av. Peter Henry Rolfss/n, Campus Universitário. 36570-000 Viçosa MG.

glaucedias@yahoo.com.br

${ }^{2}$ Departamento de

Administração,

UniversidadeFederal de

Viçosa.

${ }^{3}$ Fundação Educacional da

Serra dos Órgãos.
Abstract The importance of health systems and services evaluation has been evidenced in several discussions on policies for health and practices in services. Thus, this study was carried out to analyze the primary care to the pregnant women by the Family Health Program (PSF) in the municipality of Teixeiras, M inas Gerais State. According to the propositions by D onabedian (1990) who relates the dimensions of structure, process and result, a specific score system was used to analyze these dimensions and their respective attributes, classifying the program into the inci pient, intermediate and advanced sceneries, if the following were obtained: from 0.0 to $33.3 \%, 33.4 \%$ to $66.6,66.7$, and $100.00 \%$, respectively. In summary, such dimensions resulted into a intermediate scenery $(52.2 \%)$ for PSF characterized by an attention to the fragmented pregnant woman's health, although presenting some progresses concerning the organization of attention toward this group. Although progresses in implantation of PSF, the scenario reveals the program still presents strong characteristic marks of the hegemonic model centered on procedures and biomedical attention. Key words $\mathrm{H}$ ealth evaluation, Family $\mathrm{H}$ ealth Program, Pregnant woman's health
Resumo A avaliação em sistemas eserviços de saúdevem sendo evidenciada em diversos momentos da discussão das políticas de saúde e práticas em serviços. Desta forma, o objetivo deste estudo foi analisar os cuidados primáriosem saúde prestados às gestantes pelo Programa Saúde da Família (PSF) no município de Teixeiras (M G). De acordo com as proposições de Donabedian (1990), que relaciona as dimensões de estrutura, processo e resultado, utilizou-se um sistema de escores específicos para análisedetais dimensões eseus respectivos atributos, classificando o município nos cenários incipiente, intermediário e avançado se obtivesse entre 0,0 e $33,3 \%$; 33,4 e $66,6 \%$; 66,7 e $100,00 \%$, respectivamente. As dimensões, na síntese, resultaram em um cenário intermediário $(52,2 \%)$ para o PSF, caracterizado por uma atenção à saúde da gestante fragmentada, masque possui alguns avanços na organização da atenção para este grupo. Apesar de tais avanços na implantação do PSF, o cenário encontrado revela que o programa ainda apresenta fortes marcas características do modelo hegemônico centrado nos procedimentos e na atenção biomédica. Palavras-chave Avaliação em saúde, Programa Saúde da Família, Saúdeda gestante 


\section{Introdução}

A importância da avaliação em sistemas de saúde, para o contexto brasileiro, vem sendo ressaltada em diversos momentos da discussão das políticas de saúde e práticas em serviços ${ }^{1}$. Essa tendência se insere em um contexto mundial e, em alguns países, a avaliação já se constitui em uma prática institucionalizada e seus resultados contribuem para a formulação de suas políticas e práticas de saúde².

Entre os programas ou ações programáticas em saúde, a assistencial pré natal tem ocupado, historicamente, um espaço rel evante na atenção à saúde da população ${ }^{3}$. No Brasil, no final dos anos noventa, após duas décadas da instituição do Programa deAssistêncialntegral à Saúde da Mulher (PAISM ), a assistência à saúde da mulher permanece com muitas questões a serem enfrentadas. Entendendo que a não-percepção da mulher como sujeito este, um sintoma de um mal muito mais profundo, manifesto em toda e qualquer forma de discriminação - e o desconhecimento e desrespeito ao seu direito à saúde constituem o pano de fundo da má assistência. O M inistério da Saúde (MS) instituiu, em 2000, o Programa de Humanização no Pré Natal e Nascimento (PHPN), no qual o respeito a seus direitos e a perspectiva da humanização aparecem como elementos estruturadores ${ }^{4}$.

Apesar da importância do cuidado pré-natal, pesquisa realizada em 22 capitais brasileiras em 2002 demonstrou que a assistência pré natal no país é desarticulada e parcial ${ }^{5}$. D este modo, a atenção à saúde da mulher na gestação e parto permanece como um desafio tanto no que se refere à qualidade propriamente dita, quantos nos aspectos relacionados ao debate filosófico em torno do cuidado - nesteâmbito, podem ser mencionados a hospitalidade incondicional de Jacques Derrida ${ }^{6}$ e a compaixão laica ${ }^{7}$ - , ainda hoje centrado em um modelo medicalocêntrico, hospitalocêntrico e tecnocrático ${ }^{4,8}$.

Com a finalidade de reorganização da atenção básica e reformulação do modelo assistencial vigente, foi criado em 1994, pelo M S, o Programa Saúde da Família (PSF), o qual se respal da nos princípios da integralidade, da vigilância à saúde, da equidade, baseando-se no acolhimento, no cuidado à saúde e na humanização, entendidas como formas para se buscar uma atenção qualificada para a população. Com o objetivo de verificar a atuação do PSF na atenção a saúde da gestante, épertinente e oportuno a realização de estudos avaliativos da qualidade prestada de suas ações.

N este sentido, para Donabedian ${ }^{9}$, a qualidade na atenção à saúde pode ser definida como o grau em que se utilizam os meios mais desejáveis para alcançar melhoras na saúde. Segundo o referencial teórico sistêmico proposto pelo autor - a tríade estrutura, processo e resultado - , reflete exatamente a essência da qualidade da atenção à saúdee contribui para corrigir o curso do programa ou projeto ainda em andamento.

Com base nestes pressupostos, o presente estudo teve como objetivo a avaliação da atenção à saúde da gestante pelo PSF, o qual se revela como uma estratégia de (1) reorganização da atenção primária e (2) de mudança do modelo de atenção hegemônico.

\section{Métodos}

0 estudo envolveu 33 das 40 gestantes cadastradas no PSF do município de Teixeiras (M G); sete não quiseram participar do estudo ou não se encontraram no momento da visita domiciliar, e 35 dos 36 profissionais de saúde das quatro Unidades Básicas de Saúde(UBS) do município, sendo quatro médicos, quatro enfermeiros, quatro auxiliares de enfermagem e 23 agentes comunitários de saúde (ACS) (um estava afastado do serviço por licença médica) 0 município de Teixeiras se situa na zona da mata mineira, microrregião de saúde deV içosa, distando 204 quilômetros de Belo H orizonte, capital do estado.

A investigação, caracterizada como transversal, foi realizada por meio de entrevistas conduzidas pela pesquisadora, a partir deum questionário semiestruturado, elaborado pela equipe de coordenação do projeto. As entrevistas com as gestantes foram realizadas durante as visitas domiciliares e as entrevistas com os profissionais de saúde, nas UBS; em ambos os casos, o tempo de duração do encontro foi de trinta a quarenta minutos cada.

Antes do início das entrevistas, respeitando os aspectos éticos conformes à Resolução n 196/96 do Conselho N acional de Saúde, o projeto de pesquisa foi submetido e aprovado ao Comitê de Ética em Pesquisa com Seres Humanos da Universidade Federal de $V$ içosa. As gestantes e os profissionais de saúde assinaram um termo de consentimento livre esclarecido com todas as informações sobre 0 estudo.

A construção do model o para avaliação da atenção à saúde da gestante pelo PSF foi inspirada nas proposições de Donabedian ${ }^{9}$, que relaciona as dimensões de estrutura, processo e resultado, conforme apresentado pela Figura 1. A estrutura refere-se aos recursos utilizados pelo serviço na atenção à saúde da gestante e analisaram-se os seguin- 
tes atributos: instalação física, recursos materiais e normas. 0 processo que corresponde ao conjunto deatividades desenvolvidas na relação entre profissionais e usuários possui como atributos a qualidade do cuidado (avaliação clínica, análise do cartão da gestante e atividades educativas), a intersetorialidade, a referência e contra- referência e participação da comunidade. Já a dimensão de resultados relacionados à satisfação do usuário foi baseada nos atributos de acolhimento (sala de espera e recepção), acessibilidade (marcação de consultas, tempo de espera e barreiras geográficas) e resolubilidade (se a implantação do PSF trouxe benefícios para a saúde).

A fim de obter a classificação do PSF, das dimensões e de seus respectivos atributos, atribuiuse uma pontuação para cada critério, levando em consideração as relações hierárquicas entre os fatores que potencial mente contribuíram para a de terminação destes ${ }^{10-12}$.

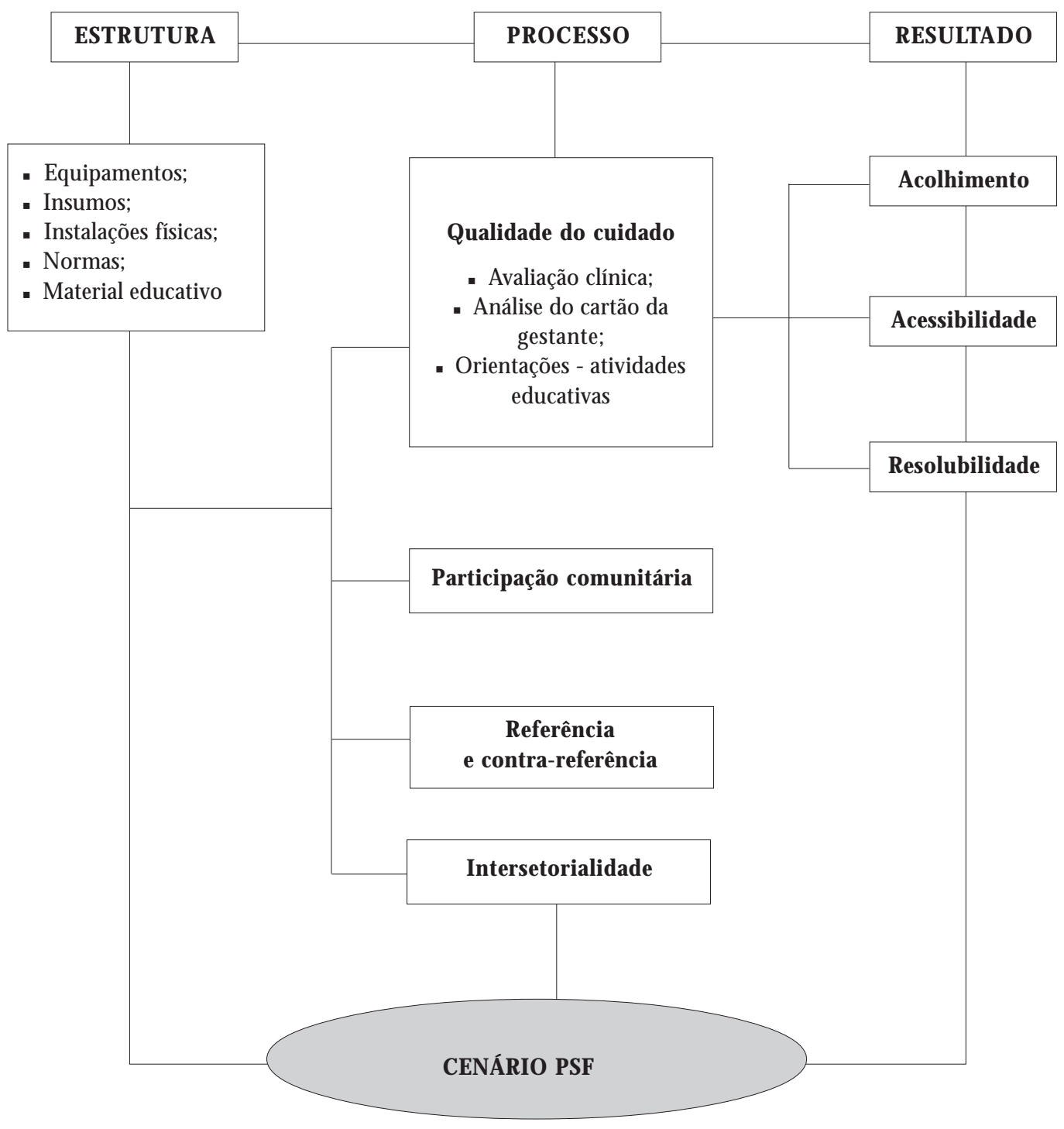

Figura 1. Desenho metodológico do estudo: atributos analisados em cada dimensão. 
Foram atribuídos pesos diferenciados para cada atributo de acordo com a sua relevância para determinada ação e dimensão. Posteriormente, 0 critério (atributo, dimensão) foi classificado como incipiente, intermediário ou avançado, se obtivesse entre 0,0 e $33,3 \%$; 33,4 e $66,6 \% ; 66,7$ e $100,00 \%$, respectivamente. Os pontos de cada indicador foram somados e depois relacionados com o máximo de pontos para a formação do escore. Os dados foram tabulados no Excel 6.0 e SPSS 10.0, o que permitiu a formação dos escores e elaboração do diagrama e das frequências.

Por fim, analisou-se a atuação do PSF nas dimensões de estrutura, processo e resultado, contextualizando-se distintos cenários que foram adaptados de Copque e $\operatorname{Trad}^{13}$. Em um cenário de implementação incipiente, a proposta aproximase da concepção de um programa que oferta uma atenção simplificada à saúde da gestante; em um cenário intermediário, observa-se uma atenção fragmentada, mas com alguns avanços; e, finalmente, em um cenário avançado, o PSF configurar-se-ia como uma estratégia de reorganização de um dos níveis de complexidade do sistema - a atenção básica - , e de mudança do modelo de aten ção hegemônico, conformeideal izado pelo Sistema Ú nico de Saúde.

\section{Resultados ediscussão}

\section{Caracterização da população estudada}

Verificou-se quea média de idade foi de $24 \pm 7$ anos (média + desvio padrão), com 33,3\% na faixa etária de 20-25 anos. As adolescentes corresponderam $27,3 \%$ da população estudada e 30,3\% encontravam-se na faixa acima dos 30 anos deidade. Eram donas de casa em sua maioria (84,8\%). $54,5 \%$ eram casadas, $27,3 \%$ apresentavam em comunhão livre e 18,2\% eram solteiras.

Com relação à renda familiar, a mediana foi de $\mathrm{R} \$ 300,00$; quanto à escolaridade, $15,2 \%$ das gestantes possuíam o ensino fundamental completo (1a a 8 a série), e 63,6\% possuíam o ensino fundamental incompleto, sendo que destas $36,4 \%$, apenas de 1 a a 4 a série. Somente $9,1 \%$ tinham o ensino superior.

Os profissionais de saúde eram em sua maioria do sexo feminino ( $74,3 \%$ ) e $82,9 \%$ residiam no município em estudo. Oitenta por cento dos profissionais foram admitidos por indicação, sendo que somente $20 \%$ passaram por um processo seletivo com análise de currículo. Ressalta-se a grande - e espúria - influência política na ocupação de cargos públicos, o que acaba por permitir a contratação de profissionais que não possuem perfil para atuação no PSF.

Avaliação das dimensões

A classificação das dimensões deestrutura, processo e resultado e seus respectivos atributos está descrita no Gráfico 1.

\section{Estrutura}

A análise da estrutura foi subdivida em instalações físicas, recursos materiais e normas existentes para as diferentes ações de atenção à saúde da gestante. As instalações físicas apresentaram-se com uma grande deficiência de espaços. Três UBS funcionavam em um mesmo local, sendo que somente uma (unidade quatro) tinha um local próprio de funcionamento. As unidades não possuíam salas suficientes e os profissionais se alternavam na realização dos atendimentos. Não havia local apropriado para atividades de educação em saúde. As instalações físicas eram precárias, velhas e com muitas dificuldades dehigienização ebiossegurança ${ }^{14}$. Não tinham banheiros separados para os profissionais de saúde, nem pia para higiene das mãos nas salas de atendimento e consultórios. Portanto, a análise da estrutura referente às instalações físicas foi considerada incipiente $(25,0 \%)$ nas unidades um, dois e três e intermediária $(41,7 \%)$ na unidade quatro,

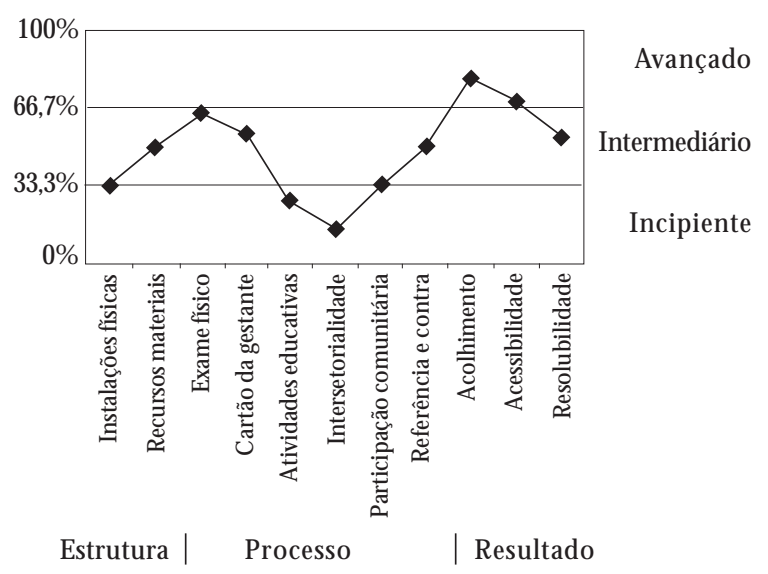

Gráfico 1. Classificação das dimensões de estrutura, processo e resultado e seus respectivos atributos, na atenção à saúde da gestante pelo PSF, município de Teixeiras (M G), 2005-2006. 
sendo quea instalação física foi considerada incipiente(33,3\%) na média dasU BS do município, conforme pode-se observar no Gráfico 1.

Com relação aos recursos materiais, apesar da escassez, existia um aporte médio segundo os critérios definidos pela OPAS ${ }^{15}$ na atenção à saúde da gestante, como a presença de estetoscópio de Pinard, mesa para exame ginecológico com banco giratório, tensiômetro revelando uma análise intermediária (50\%). No entanto, observa-se que as principais deficiências estavam na ausência de protocolos enormas das ati vidades de grupo e deeducação em saúde.

Em estudo realizado por Silveira et al. ${ }^{16}$, os serviços de atendimento pré-natal foram considerados precários, sendo a área com pior resultado a planta física, corroborando com os dados encontrados em nosso estudo.

O desenho da avaliação total da estrutura do PSF na atenção à saúde da gestante configurou-se como intermediário (46,8\%). Ao considerar asinstalações físicas, isoladamente, estas configuraramse como incipientes (Gráfico 1).

\section{Processo}

A dimensão de processo foi classificada como intermediária $(47,5 \%)$ na análise dos atributos (Gráfico 1).

A qualidade do cuidado no pré natal, que foi avaliada a partir da perspectiva da gestante e dos profissionais de saúde, correspondeu a uma análise das práticas de saúde direcionadas à atenção à saúde da gestante pelo PSF, sendo, portanto, práticas curativas, preventivas e de promoção da saúde. Buscou-se verificar os conhecimentos e atuação dos profissionais no cuidado à saúde da gestante, bem como analisaram-se as perspectivas destas frente as diferentes ações: exame físico, cartão da gestante e atividades educativas. As ações estão representadas na Tabela 1, que apresenta a classificação de acordo com as perspectivas das gestantes e profissionais.
A avaliação clínica, realizada pelos médicos, foi considerada como uma classificação intermediária $(63,9 \%)$, sendo este, dentre os aspectos do atributo qualidade do cuidado, com a maior classificação. Foram considerados os seguintes critérios da avaliação clínica: peso, aferição da pressão arterial, ausculta de batimentos cardiofetais, altura uterina, edema, exame da mama ${ }^{17}$, sendo escolhidos por enten dêlos como padrões mínimos esperados para 0 atendimento em atenção primária. De acordo com as gestantes, o peso e a aferição da pressão arterial foram avaliados em todas as consultas de acordo com o relato das 33 gestantes $(100 \%)$. Já a altura uterina e ausculta fetal foram verificadas nas consultas com menos intensidade, $40 \%$ e $44,7 \%$, respectivamente. A medida da altura uterina é um procedimento importantíssimo para acompanhamento do crescimento fetal e para determinação ou confirmação da idadegestacional ${ }^{18}$.

De acordo com as gestantes, o exame da mama e a verificação de edema não são procedimentos de rotina dos médicos, sendo que somente $28,1 \%$ das gestantes disseram queo médico realizava o exame da palpação da mama e, destas, somente duas gestantes $(9,1 \%)$ disseram que o médico realizou em todas as consultas. Nenhuma gestante relatou ter sido examinada quanto à presença de edema. $\mathrm{A}$ pesquisa de edema éextremamentenecessária para o diagnóstico e monitoramento da doença hipertensiva específica da gestação, que tantas alterações orgânicas e funcionais causa à gestante e ao concepto ${ }^{19}$.

A análise do cartão da gestante apresentou-se com uma classificação intermediária $(55,1 \%)$. O bservou-se que os cartões de acompanhamento da saúde da gestante encontravam-se incompletos, possuíam anotações relacionadas aos exames físicos elaboratoriais; no entanto, os antecedentes obstétricos, as curvas de altura uterina e de peso da gestante não estavam preenchidos em nenhuma das 34 gestantes. Isto é preocupante, pois através das curvas de peso podem ser identificados riscos relacionados com ganho de peso menor ou

Tabela 1. Classificação da qualidade do cuidado a atenção à saúde da gestante pelo PSF no município de Teixeiras (M G), 2005-2006.

\begin{tabular}{lcccc}
\hline Qualidade do cuidado: ações & Total de pontos & Total máximo & $\%$ & Classificação \\
\hline Avaliação clínica & 253 & 396 & $63,9 \%$ & Intermediário \\
Cartão da gestante & 472 & 856 & $55,1 \%$ & Intermediário \\
Atividade educativas & 105 & 408 & $25,7 \%$ & Incipiente \\
\hline
\end{tabular}


excessivo para a idade gestacional eorientar as condutas adequadas para cada caso, evitando-se resultados perinatais ruins que estejam associados ao peso materno prégravídico insuficiente, baixa estatura da mãe e aumento de peso insuficiente ou excessivo durante a gravidez ${ }^{20}$. Q uanto aos exames laboratoriais solicitados, preconizados pelo $\mathrm{M} \mathrm{S}^{17}$, a maioria ( $89,7 \%$ ) estava completo no cartão, sendo os principais: tipo sanguíneo, hemoglobina, VDRL e EAS (elementos anormais e sedimentoscopia = sumário de urina).

Com relação às orientações e atividades educativas, tanto no âmbito individual como coletivo, verificou-se uma classificação incipiente $(25,7 \%)$. Foram consideradas no âmbito individual as orientações relacionadas ao aleitamento materno, a alimentação da gestante, a imunização, as informações de preparação para o parto, cuidados com o recém-nascido, ao planejamento familiar e, no âmbito coletivo, a existência de grupos, dramatizações e outras dinâmicas.

Somente $17,7 \%$ das gestantes disseram ter sido orientadas quanto à vacinação, $23,5 \%$ foram orientadas com relação aos cuidados com o recémnascido e $41,2 \%$ disseram que o médico fazia pre paração para o parto, no entanto, abordando apenas aspectos relacionados ao tipo de parto que a gestante gostaria defazer, parto normal ou cesariana. Quanto aos aspectos alimentares na gestação e os relacionados ao aleitamento materno, somente $14,7 \%$ foram orientadas com relação ao al eitamento materno e 35,3\% foram orientadas com relação a sua alimentação no período de gestação e deamamentação. Quando questionadas sobre a alimentação durante os meses de gestação e no período de lactação e sobre o processo de amamentação, relataram tabus, dúvidas e falta de informação, podendo com os maus hábitos prejudicar a saúde do bebêedesi mesmas. 0 planejamento familiar direcionado pelo PSF, de acordo com as gestantes, foi inexistente; havia apenas uma ação individual na recomendação do uso de métodos anticoncepcionais pelo médico, não incluindo o parceiro nas discussões e decisões relacionadas ao ato de planejar. Isto foi confirmado pelos profissionais de saúde: somente $23,1 \%$ disseram realizar al guma ação relacionada ao planejamento familiar.

No âmbito coletivo, a única ação existente foram as palestras, que de acordo com os profissionais de saúde, funcionavam somenteem uma equipe (equipe um), sendo esporádicas e pouco participativas.

Percebeu-se uma relação entre o profissional médico e usuárias centrada nos procedimentos ${ }^{8}$. As consultas eram ligeiras, não possibilitando que as gestantes relatassem suas dúvidas, angústias e sofrimentos, dificultando a compreensão dos múltiplos significados da gestação e dificultando a compreensão e apreensão das gestantes de questões relacionadas à prevenção de doenças e promoção da saúde capazes de gerar qualidade de vida durante e após o prénatal.

Conforme nos relata o $\mathrm{M} \mathrm{S}^{17}$, o diálogo franco, a sensibilidadee a capacidade de percepção dequem acompanha o pré natal são condições básicas para que o saber em saúde seja colocado à disposição da mulher e da sua família - atores principais da gestação e do parto. U ma escuta aberta, sem julgamentos ou preconceitos, que permita à mulher falar de sua intimidade com segurança, fortalece a gestanteno seu caminho atéo parto eajuda a construir o conhecimento sobre si mesma, contribuindo para um nascimento tranquilo e saudável do bebê e manutenção da saúde da mãe.

Atualmente, a discussão sobrehumanização da atenção à saúde da mulher e do parto revela a autonomia e direitos como principais aspectos e já faz parte de uma série de instâncias. Entretanto, 0 quese percebeéque, na assistência pré-natal, ocorre uma expropriação da autonomia da mulher, se centrando no modelo tecnocrático em que as muIheres são vistas como objeto, fora de contexto, em quea máquina é examinada e não se estabelece nenhum vínculo com o profissional ${ }^{4}$. E como achado de nosso estudo, verificou-se que as ações praticadas foram de responsabilidade dos profissionais que supervalorizavam as mais técnicas e mantêm, com intervenções determinadas, a hierarquia e padronização de rotinas.

Através disso, observou-se que, apesar do PSF se propor a uma relação diferenciada com os seus usuários e com a comunidade, principalmente no que se refere à vigilância em saúde, nas formas de prevenção e promoção, muito pouco tem sido feito nestes aspectos, pois os profissionais de saúde continuam atuando numa percepção biologista e fragmentada do ser humano, no caso das gestantes, atuando meramente através de consultas-procedimento, sendo, portanto, a qualidade do cuidado classificada como intermediária (50\%).

A fim de aprofundar os caminhos de reformulação do modelo assistencial e reorganização da atenção básica apontados pelo PSF, avaliamos atributos organizativos e interativos que respaldam e viabilizam as práticas e ações de atenção à gestante, sendo eles: a participação comunitária, a referência e contra-referência ea intersetorialidade.

A avaliação da participação comunitária nas atividades deeducação em saúde, desenvolvidas pelo PSF, revel ou a pouca inserção das gestantes nas ati- 
vidades educativas, considerando participação em grupos, palestras e dramatizações. Este atributo caracterizou-secomo incipiente (33,3\%) (Tabela2).

0 reconhecimento desujeitos está no centro de todas as propostas renovadoras identificadas no setor saúde, inclusive no PSF. Realmente, pode-se perceber que, a partir dos objetivos do programa (humanização das práticas; democratização do conhecimento; desenvolvimento da cidadania; estimulação da organização da comunidade), há uma val orização dos sujeitos e de sua participação nas atividades desenvolvidas pelas UBS, bem como a resolutividade dos problemas da comunidade $e^{21}$. No entanto, não é isso que verificamos em nosso estudo. O PSF não integrava as gestantes na formulação de suas atividades, muito menos promovia o desenvolvimento da cidadania.

Conforme nos relata Guizardi et al. ${ }^{22}$, construir caminhos para a participação no SU S não implica apenas melhor informar ou capacitar a população e seus representantes. [...] A partici pação como princi pio de constituição das políticas públicas só se concretiza quando vozes diversas se apresentam ao diálogo como sujeitos na construção de sentidos e mundos. Pensar a participação significa pensar esta implicação política que inclui espaços já institucionalizados, eo PSF éum destes espaços, mas não se esgota neles.

O bservou-se uma grande dificuldade dos profissionais de saúde do município na execução das atividades educativas para o grupo materno; as poucas iniciativas etentativas foram frustradas pela pouca participação das mães nas atividades desenvolvidas. No entanto, as gestantes relataram em sua maioria ( $72 \%$ ) a inexistência ou desconhecimento de atividades educativas no PSF.

As estratégias educacionais e comportamentais visam aumentar a consciência sobre os riscos e sobre a importância de comportamentos seguros, além de oferecer incentivos e modelos positivos. No entanto, o que se pode perceber a despeito das práticas de educação em saúde é que a elaboração das atividades está ainda fundamentada no modelo tradicional, em que os usuários são tomados como indivíduos carentes de informação em saúde. Desta forma, a comunicação dos profissionais de saúde assume uma atitude paternalista, no que fazer e como fazer para a manutenção da saúde. Freire ${ }^{23}$ revela: "saber ensinar não é transferir conhecimento, mas criar possibilidades para sua própria produção ou a sua construção".

Para análise da referência e contra-referência, foi considerada a integração do PSF com os hospitais que possuem maternidade na microrregião. A avaliação nos apontou para uma classificação intermediária (50\%), conforme apresentado pela Tabela 2.

Os profissionais de saúde declararam uma maior facilidade de referência. A assistência ao parto era realizada nos municípios vizinhos. Todavia, os profissionais relataram grandes dificuldades no retorno dasinformações, na contra-referência. N ão havia relatório por escrito, no processo de contrareferência, os obstetras não davam nenhum tipo de retorno ao médico da atenção básica, muito menos aos enfermeiros e outros profissionais do PSF, normalmente a informação era proveniente da própria gestante. Isto dificulta a integralidade, que é um dos princípios básicos do SUS.

A ausência de informação revelou a falta de integração entre os hospitais e os serviços de assistência pré-natal, impedindo que o processo reprodutivo seja visto em sua totalidade, favorecendo descontinuidade na assistência à saúde do binômio mãe-filho, culminando com maior risco de morte da mulher e da criança ${ }^{24}$.

As gestantes relataram um grande sentimento de insegurança ao fazer o pré- natal com um médico e ser encaminhada para outra cidade e realizar o parto com outro médico. Caso a referência e contra-referência funcionassem adequadamente, 0 problema poderia ser amenizado. Segundo Serruya et al. ${ }^{4}$, através de seu estudo sobre o panorama da atenção ao pré-natal no Brasil, a questão da

Tabela 2. Classificação dos atributos intersetorialidade, referência e contra-referência e participação comunitária, pertencentes à dimensão de processo - avaliação do PSF na atenção à saúde da gestante no município de Teixeiras (M G), 2005-2006.

\begin{tabular}{lcccl}
\hline \multicolumn{1}{c}{ Atributos } & Total de pontos & Total máximo & $\%$ & Classificação \\
\hline Participação comunitária & 44 & 132 & $33,3 \%$ & Incipiente \\
Referência e contra-referência & 16 & 32 & $50,0 \%$ & Intermediário \\
Intersetorialidade & 10 & 72 & $13,9 \%$ & Incipiente \\
\hline
\end{tabular}


falta de vínculo entre assistência pré-natal e a do parto leva as mulheres, em trabalho de parto, a uma peregrinação à procura de vagas nos hospitais. Além disso, retrata que a maioria das mortes maternas ocorre perto do parto, demandando intervenções que garantam melhor assistência nesse período. N esse panorama da situação obstétrica, a existência da desumanização em um momento tão importante eo direito que toda mulher tem de garantia ao atendimento são questões emblemáticas a serem enfrentadas.

M elleiro et al. ${ }^{25}$ apontam em seu estudo que a falta de integração entre os serviços de saúde, além de acarretar uma ruptura das ações iniciadas nas UBS, durante o pré-natal, tornava a procura pela instituição hospitalar, por ocasião do parto, fonte de ansiedade e risco, com repercussões negativas para o processo de nascimento.

De acordo com a proposta da Equipe de Referência eapoio $M$ atricial do $M S^{26}$, énecessário quea equipe tenha um contato direto com os especialistas e estes com a equipe, pois é preciso enxergar 0 usuário como um todo (inserção social, situação afetiva, outros problemas orgânicos). Isto garante queno conjunto pré natal e puerpério ocorra mais benefícios do que danos para a gestante e que 0 projeto deacompanhamento envolva um compromisso maior com o usuário.

De forma complementar, analisou-se também a intersetorialidade, verificando se havia ou não parcerias e/ou integração do PSF com outros setores do município: educacional, ambiental, assistência social, etc. N esta perspectiva, a avaliação deste atributo foi considerada incipiente $(13,8 \%)$.

Ferreira ${ }^{27}$ revela que as ações intersetoriais de seu estudo também encontravam-se em um estágio incipiente de implantação e quea Conferencia N acional de Saúde em 2001 revelou o sistema de saúde no Brasil fragmentado e a intersetorialidade precária.

Os profissionais de saúde do município de Teixeiras, quando questionados sobre a ausência ou o pouco fomento de ações intersetoriais, manifesta- ram um desconhecimento do que seja intersetorialidade, revelando uma falta de preparo dos recursos humanos. Estes achados corroboram os estudos de Junqueira ${ }^{28}$, que apresentam as dificuldades para a intersetorialidadeem três categorias: relacionadas ao tipo de gestão, especialmente nas formas de financiamento que são setorializadas, gerando atraso na liberação de recursos; relacionadas à ausência de cultura da intersetorialidade, que prioriza interesses particulares acima dos interesses construídos coletivamente; e relacionadas à falta de preparo dos recursos humanos, que centram esforços na organização do modelo médico-assistencial.

J unqueira ${ }^{29}$ ressalta a importância da intersetorialidade, revelando que a qualidade de vida passa pela interação de várias dimensões do bem-estar físico, psíquico e social, e demanda visão integrada dos problemas sociais. N este sentido, as políticas setoriais isoladamente são incapazes de considerar o cidadão na sua totalidade e nas suas necessidades individuais e coletivas.

\section{Resultado}

A avaliação da dimensão de resultado foi realizada a partir dos atributos acessibilidade, acolhimento e resolubilidade, conforme nos apresenta a Tabela 3, na perspectiva das gestantes. A classificação que se configurou ao analisar os três atributos foi avançada (69,3\%). É importante destacar que os atributos se relacionam e, portanto, devem ser analisados como um todo.

A avaliação do acolhimento isoladamente re sultou em uma classificação avançada (79,4\%). A maioria das gestantes se sentia bem acolhida na recepção das U BS. No discurso, estavam presentes relatos relacionados à educação, à atenção e à simpatia, enfatizando-seo "mel hor atendimento quando comparado ao hospital". Os ACS também realizavam, de acordo com os depoimentos das gestantes, um papel essencial, ao acolher as informações, dificuldades, necessidades das gestantes e de seus familiares.

Tabela 3. Classificação dos atributos resolubilidade, acessibilidade e acolhimento pertencentes à dimensão de resultado - avaliação do PSF na atenção à saúde da gestante, município de Teixeiras (M G), 2005-2006.

\begin{tabular}{lcccc}
\hline \multicolumn{1}{c}{ Atributos } & Total de pontos & Total máximo & $\%$ & Classificação \\
\hline Acolhimento & 154 & 194 & $79,4 \%$ & Intermediário \\
Acessibilidade & 135 & 194 & $69,6 \%$ & Intermediário \\
Resolubilidade & 70 & 130 & $53,8 \%$ & Intermediário \\
\hline
\end{tabular}


As atividades de recepção na U BS eram realizadas pelas recepcionistas, que seguiam uma agenda com consultas pré-marcadas pelos ACS. No caso das gestantes, havia uma maior facilidade na marcação de consultas; por ser uma determinação do MS que ela tenha no mínimo seis consultas, a gestante já saia da UBS com sua consulta marcada para o outro mês. A única reclamação presenteno depoimento das gestantes foi quanto à fila de espera, as consultas eram realizadas obedecendo ao critério da ordem de chegada pelos usuários. D evese destacar que este é um critério de recepção excludente e criticado pelo MS no programa de humanização da aten ção à saúde ${ }^{26}$.

O utro aspecto a ser destacado refere-se ao fato de que 14 gestantes $(42,4 \%)$ realizavam o pré-natal no hospital, dando preferência ao especialista. Observou-se, portanto, que o PSF nem sempre funcionava como porta de entrada para o sistema de saúde, ou seja, nem todas as mães procuravam o PSF para fazer o pré-natal. Em estudo realizado por Nagahama et al. ${ }^{5}$, no município de Maringá (PR), 78\% das gestantes deslocaram-se do posto de saúde perto de sua residência na busca de qualidade técnica e humana.

Não obstante, as gestantes se sentiam bem acoIhidas e conseguiam diferenciar o PSF do hospital quando relatavam uma melhor recepção do PSF do que do hospital, e também enfatizavam o papel do ACS nas visitas domiciliares, ouvindo suas queixase permitindo que expressassem suas preocupações.

Quanto à acessibilidade, foram considerados o tempo de espera, a marcação de consulta e as barreiras geográficas como critérios de análise do atributo acessibilidade. Em relação à acessibilidade, esta configurou-se em um atributo avançado $(69,6 \%)$ (Tabela 3).

A maior dificuldade quanto à acessibilidadefoi à geográfica, especialmente para as gestantes residentes na zona rural. $42 \%$ das entrevistadas residiam nazona rural eapresentaram suas queixas princi palmente relacionadas à falta de transporte e dificuldades de acesso às consultas em períodos de chuva. Vale ressaltar que as opções de transporte destas gestantes, ou seja, o serviço de transporte coletivo precário, o transporte por bicicletas, carroças e cavalos, faziam com que essas mulheres gastassem nestepercurso horas de seu tempo, além de dinheiro e de implicar cansaço físico, psicológico e riscos à saúde.

De toda forma, as gestantes informaram que estavam satisfeitas com a marcação da consulta, podendo ser realizada pelo ACS ou pela UBS, garantindo acesso ao atendimento e facilitando a obtenção da consulta, já que antes do PSF era ne- cessário acordar de madrugada e enfrentar fila no hospital. 0 tempo de espera para 0 atendimento na UBS foi a maior reclamação das gestantes, que relataram precisar chegar cedo para serem atendidas com menos demora, corroborando com os achados de Tsunechiro et al. ${ }^{30}$, na cidade de São Paulo, os quais evidenciam a longa espera das gestantes para serem atendidas, aguardando horas a sua vez, o que pode justificar a procura das gestantes por especialista no hospital.

No que se refere à resolubilidade, foram analisados aspectos relacionados à percepção das gestantes do lugar do PSF no contexto de organização da saúde no município, ou seja, com a implantação do PSF, houve melhoria na atenção sanitária do município?A resolubilidade apresentou-secomo um atributo intermediário (53,8\%) (Tabela 3).

As gestantes destacaram no atendimento do PSF as visitas domiciliares realizadas pelos ACS, assim como do médico e enfermeiros, o cuidado com os idosos, hipertensos e diabéticos ea entrega de medicamentos e exames. Contudo, as gestantes relataram dificuldades e limitações específicas do programa, reduzindo o percentual de classificação para intermediário, retrataram aspectos ligados à falta de informação, à exiguidade das práticas educativas por parte dos profissionais de saúde e a falta de integração entre os médicos - aquele que real iza o pré-natal e aquele que real iza a parturição (obstetra) - , sendo este um aspecto que gera insegurança e apreensão na realização do parto.

Estudo realizado por Brienza et al. ${ }^{31}$, que avalia o acesso ao prénnatal à rede básica do município de Ribeirão Preto, demonstra que o modelo de assistência evidenciado pelas mulheres reforça a visão de uma assistência pré-natal voltada para 0 cuidado físico e biológico, referindo-se a um mesmo ritual: peso, medição e pressão.

Desta forma, pese a que as usuárias gestantes relatem certa satisfação com o acesso ao serviço de saúde, observou-se que apenas o acesso é insuficiente na promoção de sua saúde e na saúde do seu bebê, é preciso fundamentar as ações em certas atitudes como a cultura do diálogo, da escuta ativa, da informação, inserindo-se no processo e desenvolvimento de práticas mais humanas e resolutivas.

\section{Conclusões}

O cenário produzido na atenção a saúde da gestante pelo PSF foi intermediário (52,2\%), caracterizado pela fragmentação de suas ações, mas sendo perceptíveis alguns progressos na sua implementação. Avanços relacionados à adesão das gestantes à 
proposta do PSF - na realização das visitas domiciliares, no acolhimento - a maior facilidade de acesso proporcionada pela presença do ACS, na marcação de consultas, são bons exemplos disto.

$\mathrm{N}$ a síntese das dimensões, a falta de adequado espaço físico - o que interfere na realização das atividades assistenciais e educativas - , bem como a inexistência da participação comunitária, da intersetorialidade e da contra-referência, afetam as ações de saúde, desintegrando as partes e permanecendo 0 ol har reducionista da biomedicina. Torna-se, assim, perceptível, a necessidade de se evoluir para um tratamento mais complexo - ecompleto - da organização dos serviços desaúde, uma vez que, de fato, as poucas atividades preventivas e de promoção de saúde do PSF demonstram que o programa, da forma como se apresenta atualmente, funciona mais como um suporte para o hospital, sendo mais um local de atendimento procedimento-centrado, dificultando a assimilação de uma atenção usuária - centrada em prol da reorientação do modelo assistencial.

Destaca-se a premência de se investir nos recursos humanos em três aspectos fundamentais: o primeiro se refere à atuação técnica, o segundo, ao aspecto relacional dos profissionais de saúde com os usuários, superando uma abordagem me- ramente biológica para uma abordagem integral, holística e complexa das gestantes e o terceiro, à forma de contratação dos profissionais. Os atributos de participação comunitária, intersetorialidade, referência econtra-referência devem ser prioridades dos gestores, permitindo e garantindo a transformação do modelo assistencial hegemônico vigente, na medida em que consideram o ser humano na sua totalidadeena sua complexidade, possibilitando a construção de novos conceitos e novas formas de agir em saúde.

Por fim, verifica-se que melhores resultados podem ser alcançados pelo PSF, uma vez que a inserção das usuárias (gestantes) na formulação das ações soma esforços em favor de uma prática de atenção perinatal mais humana e cuidadora. Ao valorizar os aspectos relacionais, as gestantes consideram que a atenção deve ser voltada para uma abordagem que as perceba em sua totalidade, enfatizando a necessidade de um maior vínculo com os profissionais de saúde. N esta perspectiva, caminhos podem ser traçados rumo à reformulação do sistema de saúde hegemônico e vigente, de modo a torná-lo, quiçá, mais orientado para o amparo dos diferentes sujeitos que compõem a (complexa) realidade em prol de um cuidado em saúde mais humanizado e solidário.

\section{Colaboradores}

GD Costa participou da idealização, delineamento de objeto de estudo, desenho da metodologia, coleta e análise dos dados e redação do artigo; RM M Cotta participou da idealização, delineamento de objeto de estudo, desenho da metodologia e foi responsável pela coordenação, orientação e acompanhamento do trabalho de campo, da redação e revisão do artigo; JR Reis e SCC Franceschini participaram do planejamento, orientação técnica e acompanhamento do trabalho; R Siqueira-Batista e AP Gomes participaram da redação do artigo e sua revisão crítica. 
1. Carvalho DS, Novaes HMD. Avaliação da implantação de programa de atenção pré-natal no município de Curitiba, Paraná, Brasil: estudo em coorte de primigestas. Cad Saude Publica 2004; 20(2):S220-230.

2. Hartz ZM A. Institucionalizing the evaluation of health programs and policies in France: cuisine internationale over fast food and sur measure over ready made. Cad Saude Publica 1999; 15(2):229-259.

3. D'Oliveira AFPL, Senna DM. Saúde da mulher. In: Schraiber LB, N emes MIB, Gonçalves RBM, organizadores. Saúde do adulto: programas e ações na unidade básica. São Paulo: Hucitec; 1996. p. 86-108.

4. Serruya SJ, Cecatti JG, Lago TG. O Programa de Humanização no Pré $\mathrm{N}$ atal e Nascimento do M inistério da Saúde no Brasil: resultados iniciais. Cad Saude Publica 2004; 20(5):1281-1289.

5. Nagahama EEI, Santiago SM. O cuidado pré natal em hospital universitário: uma avaliação de processo. Cad Saude Publica 2006; 22(1):173-179.

6. Derrida J. Voyous. Paris: Galilée; 2003.

7. Siqueira-Batista R. Bioética e compaixão. Jornal do Cremerj 2004; 17(1):15.

8. Merhy EM . Introdução ao debate sobre os componentes da caixa de ferramentas dos gestores em saúde. Ver SUS/Brasil - Vivências e estágios na realidade do SUS do Brasil. 2002. [acessado 2006 jan 28]. Disponível em: http://dtr2002.saude.gov.br/versus/index.html

9. Donabedian A. Garantía y monitoria de la calidad de la atención médica: un estudio introductorio. M éxico: Instituto Nacional de Salud Pública; 1990.

10. Frias PG, Lira PIC, Hartz ZM A. Avaliação da implantação de um projeto para redução da mortalidade infantil. In: Hartz ZM, Silva LM V, organizadores. Avaliação em saúde: dos modelos teóricos à pratica de programas e sistemas de saúde. Rio de Janeiro: Fiocruz; 2005. p.151-191.

11. Felisberto E, Carvalho, EF, M aggi RS, Samico I. Avaliação do processo de implantação da estratégia da Atenção Integrada às doenças prevalentes da infância no Programa Saúde da Família, no estado de Pernambuco, Brasil. Cad Saude Publica 2002; 18(6):1737-1745

12. Lopes RM, Silva LM V, Hartz ZMA. Teste de uma metodologia para avaliar a organização, acesso e qualidade técnica do cuidado na atenção à diarréia na infância. Cad Saude Publica 2004; 20(2):S283-S297.

13. Copque HLF, Trad LAB. Programa Saúde da Família: a experiência de implantação em dois Municípios da Bahia. Epidemiologia e serviços de saúde 2005; 14(4):223233.

14. Martins $M$, Siqueira-Batista $R$, Sucupira ED, Freitas LM, Brito RC, Guedes AGM, Zuim R, Bethlem EP, Gomes AP, Selig L. Recomendações da Assessoria de Pneumologia Sanitária do Estado do Rio de Janeiro para Biossegurança em Unidades Básicas de Saúde UBS. Pulmão RJ 2004; 13(3):190-194.

15. OPAS/OM S/Brasil. Condições de eficiência dos serviços de atenção materno infantil. [Mimeo 1987].

16. Silveira DS, Santos IS, Costa JS. Atenção pré-natal na rede básica: uma avaliação da estrutura e do processo. Cad Saude Publica 2001; 17(1):131-139.

17. Brasil. M inistério da Saúde. Pré-natal e puerpério: atenção qualificada e humanizada [M anual técnico Série $A$ - Direitos Sociais e reprodutivos - Caderno no 5]. Brasília: M inistério da Saúde; 2005.
18. Rezende J, M ontenegro CAB. Obstetrícia fundamental. 10a ed. Rio de Janeiro: Guanabara Koogan; 2005.

19. Federação Brasileira das Sociedades de Ginecologia e Obstetrícia. Assistência Pré Natal - Manual de Orientação. Rio de Janeiro: Federação Brasileira das Sociedades de Ginecologia e Obstetrícia; 2001.

20. Brasil. M inistério da Saúde. Assistência pré-natal: normas e manuais técnicos. Brasília : Secretaria de Políticas de Saúde/M inistério da Saúde; 2000.

21. Alves VS. Um modelo de educação em saúde para o Programa Saúde da Família: pela integralidade da atenção e reorientação do modelo assistencial. Interface (Botucatu) 2005; 15:39-52.

22. Guizardi FL, Pinheiro R, M achado FPS. Vozes da participação: espaços, resistências e o poder da informação. In: Pinheiro R, M athos RA, organizadores. Construção social da demanda. Rio de Janeiro: Abrasco; 2005. p. 303.

23. Freire P. Pedagogia da autonomia: saberes necessários a pratica educativa. 30a ed. São Paulo: Paz e Terra; 2004.

24. Tanaka AC, Siqueira AAF, Bafile PN. Situação de saúde materna e perinatal no Estado de São Paulo. Rev. Saude Publica 1989; 23:73-79.

25. M elleiro MM, Gualda DM R. Experiências e expressões de gestantes na interação com o sistema de saúde: um enfoque fotoetnográfico. Rev. Latino-am enfermagem 2004; 12(3):503-510.

26. Brasil. M inistério da Saúde. Secretaria-Executiva. Núcleo Técnico da Política Nacional de Humanização. HumanizaSU S: equipe de referência e apoio matricial/M inistério da Saúde, Secretaria-Executiva, Núcleo Técnico da Política Nacional de Humanização. Braślia: M inistério da Saúde; 2004.

27. Ferreira VSC, Silva LM V. Intersetorialidade em saúde: um estudo de caso. In: Hartz ZM A, Silva LM V. Avaliação em saúde dos modelos teóricos à pratica na avaliação de programas e sistemas de saúde. Rio de Janeiro: Fiocruz; 2005.

28. Junqueira RGPA. A intersetorialidade do ponto de vista da educação ambiental: um estudo de caso. Rev. Adm. Publ. 1998; 32(2):79-91.

29. Junqueira LAP. Novas formas de gestão na saúde: descentralização e intersetorialidade. Rev. Saúde e Sociedade 1997; 6(2):31-46.

30. Tsunechiro MA, Bonadio IC, Oliveira VM. Acolhimento: fator diferencial no cuidado pré-natal. In: Anais do 8o Simpósio Brasileiro de Comunicação em Enfermagem. [site na Internet]. [acessado 2006 jul 23]. Disponível em: htpp://www.proceedings. scielo.br/scielo.php

31. Brienza AM, Clapis MJ. Acesso ao pré-natal na rede básica de saúde do município de Ribeirão Preto: análise da assistência recebida por um grupo de mulhe res. In: Anais do 8o Simpósio Brasileiro de Comunicação em Saúde. [site na Internet]. [acessado 2006 jul 21]. Disponível em: htpp://www.proceedings. scielo.br/scielo.php

Artigo apresentado em 19/09/2006

Aprovado em 09/07/2007

Versão final apresentada em 22/08/2007 